\title{
Decoding Syrian Refugees' Covid-19 Vulnerability in Informal Tented Settlements: a Community/Refugee-Led Approach to Mitigate a Pandemic Outbreak
}

Paul Moawad, Bartlett School of Planning, University College London. paul.moawad.19@ucl.ac.uk

Lauren Andres, Bartlett School of Planning, University College London. l.andres@ucl.ac.uk

\section{Introduction}

The implications of Covid-19 surpass the health aspects and bear heavy consequences for the most vulnerable, especially those in Informal Tented Settlements (ITSs), unable to work, hence accentuating poverty and hunger. This is the case for refugees. The health crisis has led to stricter mechanisms in encampments hindering the refugees' capacity to survive. While it is still too early to evaluate the impact of the pandemic on such fragile communities, there is an urgent need to raise alerts on the impact of healthled preventive and containment measures on marginalising refugees further. This imperative builds upon recent calls to reconsider the local response to health emergencies in informal urban settlements (Wilkinson, 2020). Planners have a key role to play in addressing refugees' vulnerabilities holistically with a more inclusive understanding of their needs along with host community's members. Refugees' livelihoods vary from one context to another; attention is here given to Lebanon and specifically Syrian's refugees in ITSs.

The start of the Syrian crisis in 2011 led to an unprecedent mass influx of refugees which have disrupted the Lebanese demographics disproportionally but also changed the cityscape in increasing its urban density and the rural landscapes, replacing agricultural lands with ITSs. While the national government adopted a laissez-faire approach, the burden was shifted onto international non-governmental organisations (INGOs) and local municipalities. This led to an absence of coherent and cross-linked strategies for the inclusive development of those areas and increased strain on an already debilitated infrastructure, local economies and shelter conditions. This is particularly the case in the most remote locations like those in the Beqaa governate, an area that is central to both Beirut and Damascus.

The Lebanese government's response to the global pandemic has been praised as it has resulted in a small surge of cases and deaths despite a fragile political and economic context. A national curfew and a state of emergency were imposed, coupled with preventive measures such as airport lockdown, closure of all public outlets and restaurants and rapid public awareness. Local municipalities and security forces across Lebanon added supplementary encampment mechanisms by locking the entry and exit of all refugee camps and settlements. INGOs also developed thorough response plans based on multi-level interventions, spanning from a micro-level (household level and community level) to a macro-level (area/municipal level and full quarantine). Until May $25^{\text {th }}$, only one case in a Palestinian refugee camp was identified and contained. However, as of June $4^{\text {th }}, 15$ cases amongst Syrian refugees have been reported in Majdal Anjar in the Beqaa Governorate.

The situation can therefore rapidly deteriorate, which will have direct and indirect consequences on refugees and raises severe challenges. There is a scission between 
planned mechanisms designed to create pandemic-resilient built environments versus forced encampment measures in unplanned and informal settings where health considerations along with socio-economic needs are barely accounted for. In the context of refugee camps in remote locations, where the refugees are already frail and isolated, this is intensified. At the crux of this dilemma is the relationship between stricter healthmotivated lockdowns and increased vulnerabilities linked to decreased access to basic services, (informal) work opportunities and health support services. We argue for the need to construct more inclusive community/refugee-led responses to better link planned and unplanned health emergency responses.

This discussion rests upon the combination of two research projects: a scoping smallscale research project about the impact of the current Covid-19 related policies and strategies from March 2020 to May 2020 in refugees' ITSs coupled with a research study looking at Syrian refugees' livelihoods in remote locations conducted between October 2019 and February 2020. The latter research includes 107 interviews with refugees and 58 interviews with representatives from national governmental representatives, nonacademic and academic experts, host community members, INGOs and nongovernmental organisations (NGOs).

\section{Pre-Covid-19 Informal Tented Settlements}

To define what is informal versus the formal is difficult in the Lebanese context as the country has been affected by an ongoing influx of refugees since 1943, leading to successive waves of settlements, as well as internal displacements (Fawaz and Peillen, 2003). The UNHCR defines Lebanese ITSs as those established in an unplanned and unmanaged manner, through a direct agreement between refugees and landlords (UNHCR, 2019). The unplanned nature of recently formed Syrian ITSs is, however, not new. Pre-Syrian crisis, tents were informally set up on agricultural lands for those working in the fields (Turner, 2015) and were dismantled shortly after the spring and summer farming seasons. The massive influx of refugees changed these agricultural fields into marginalised refugee communities with poor livelihood and shelter conditions. Hence quasi-permanent vulnerability replaced what was perceived as a seasonal and temporal labour settlement in a haphazard manner. The lack of planning coordination combined with national and local laissez-faire led to the eradication of several agricultural fields. This impacted an agriculture-dependant local economy while challenging any attempts to develop regulatory land-use planning frameworks.

As economic conditions started changing in the last five years, with less job opportunities for Lebanese nationals, the informal labour market became regulated from 2017 to lessen competitiveness, only allowing Syrian refugees to work in agriculture, construction and the environment. With less informal work opportunities refugees' socioeconomic vulnerabilities deepened. It became necessary for refugees to seek work opportunities further away, stressing the importance of being mobile to survive.

Refugees' living conditions are extremely dire, making them highly vulnerable from a health perspective. Access to healthcare services is limited and complex, dependant on the rights and status of each individual refugee. As former agriculture lands, ITSs are offgrid. The use of temporal and easily dismantled materials to provide shelter does not provide adequate hygiene and disease-free environments. Rodents, reptiles and insects pose serious illness-related threats to refugees who place chicken wires around their 
tents to mitigate these non-hygienic concerns. Attempts of collection and treatment of waste and wastewater management do not meet international hygiene and safety standards, even though managed by either NGOs or subcontracted local agencies. Shared pit latrines are exposed, with foul smells from waste and black and grey water running through the trenches. The health consequences are major, aside from the potential for any pandemic crisis they also contribute to raising tensions with planned settlements leading to stigmatisation and rejection (Thorleifsson, 2016). Such stigmatisation has been reinforced by hegemonic policies and restrictive governmental measures accentuating social tensions. Since 2014, 45 municipalities nationwide have imposed curfews on Syrian refugees preventing their mobility outside the perimeter of their settlements. The Covid-19 crisis has destabilised refugees' livelihoods further by forcing supplementary control of the unplanned through forced control of encampments and additional stern curfews.

\section{Syrian refugees' encampment and mobility restrictions amidst Covid-19}

The Lebanese national response to the pandemic was aligned with international measures across the world. What differs significantly, though, is how the health crisis rational gave leverage to local municipalities to justify further lockdown mechanisms, hence strengthening the process of encampment and, correlatively, control and oppression, making informal spaces places of exception (Bakewell, 2014). Shortly after the national lockdown on 15 March 2020, 18 municipalities have introduced illegal and restrictive curfews that do not apply to Lebanese citizens but significantly constrained refugees' movements. From mid-March, the government have also imposed a new measure where only one person from each Syrian refugee settlement would be allowed to exit for the sole purpose of buying food and other goods while the rest of the settlement population is prohibited from doing so (Hodali, 2020). As a result, refugees are trapped inside their ill-constructed habitats with no prospect of access to informal jobs, which, in the context of the pandemic, led to a loss of consumer confidence, a negative growth and a severe economic contraction. The refugees are left in a new state of limbo struggling to cope and survive and make an active use of time.

Now, restrictions on mobility for refugees by a forceful lockdown on their points of entry and exit has exacerbated the social and economic burden even further inside and outside of the ITSs, congealing the boundaries of the informal and the formal. This strain is affecting vulnerable host communities that used to benefit from refugees' mobility and their commercial activities. Porous though tense, these spatial and socio-economic relationships are put at risk leading to an increased socio-spatial marginalisation and stigmatisation of refugees. The key issue is a lack of response to address both combined vulnerabilities. From the time at which the ITSs started to settle there have been no actions or steps taken by national and local governments to tackle such compounding problems. Emergency planning, humanitarian aid and supply of food services are left in the hands of humanitarian agencies that are now redistributing resources to both Syrian refugees and vulnerable host community members based on quotas.

Restrictions on mobility in the encampments also have direct consequences on refugees' health and specific Covid-19 health responses raising significant concerns if the pandemic reaches ITSs. A national Covid-19 task force between the Lebanese state, local municipalities and UN agencies has been created but attempts to implement preventive measures, such as isolation units, have been delayed due to inadequate and 
ill-equipped facilities. Though a multidimensional response plan ensuring free Covid-19 testing and treatment as well as self-imposed quarantine is in place, any attempt to provide health-oriented 'planning' initiatives is extremely difficult and complex due to the intrinsically temporary and unplanned nature of those settlements and livelihood conditions: tents and precarious structures offer limited space for large families, options for creating isolation units are scarce resting upon re-using existing and/or abandoned structures, social distancing is of course impossible not to mention any preventive measures related to water, sanitation and hygiene (WASH). As a result, containing the virus in camps has mostly relied on awareness sessions based on informing refugees about the pandemic, which has increased their fear and self-isolation.

Limited access to healthcare facilities and availability of adequate isolation units in case of an outbreak raises significant concerns for the ability to control and manage the pandemic spread inside but also outside of the settlements. The unplanned nature of the refugees' livelihoods requires an alternative 'refugee-led local response' to address the current crisis but also future pandemic outbreaks. This should be constructed in line with a thorough and holistic account of refugees' vulnerabilities and ITSs planning.

\section{Calling for community/refugee-led local responses}

'Informal settlements face considerable challenges around the control of COVID-19, but locally developed strategies could mitigate the worst of the outbreak as long as action is taken fast' (Wilkinson, 2020,16). In Lebanon, the current crisis, aligned with a decade of the country struggling with the spread of ITSs, has revealed the severe hurdles affecting any attempts to address health concerns in unplanned settings: preventive and treatment healthcare mechanisms are extremely limited and hindered by living conditions; coordination amongst national and local authorities and UN agencies is difficult, and informal sector and survival options are shrinking. Significant shifts based on addressing refugees' vulnerabilities holistically with a more inclusive understanding of their needs along with those of host communities are needed.

While the pandemic situation has been contained to date this surprising fact should not be credited to the forcefully imposed governmental lockdowns on these ITSs. It has been achieved thanks to the continuous INGOs support who have developed localised approaches putting the refugees' needs and practices first, along with the settings within which they sit. This has allowed ensuring survival and raising awareness about the pandemic. This suggests that any attempts to develop community/refugee-led local responses to tackle health concerns and future pandemics in ITSs have to address the following four pillars.

Pillar 1: Temporary social cohesion and integration can be concretised via the routes of collaborative community/refugee-led responses. Increased encampment and restriction of mobilities negatively impact both refugees and the host communities' vulnerabilities. Recognising the historic socio-economic ties between those groups rather than increasing rejection and fear forms the first step towards the development of a more inclusive living environment for all parties.

Pillar 2: Health-led design approaches, tackling both well-being and disease-free environments, can be introduced to the temporariness of such adaptable and fragile built 
environments. ITSs are characterised by their non-permanent structures though refugees attempt to recreate their home in their tents. There is a sense of ownership and permanence in the unplanned nature of those settlements, which can be used to implement micro-level changes in refugees' living spaces, while empowering them in delivering them.

Pillar 3: Multidimensional awareness has to be sustained. Providing information to refugees has been one of the most efficient actions to date to contain the pandemic. This testifies to the importance of putting refugees in the centre of preventive actions rather than the recipient of those actions, hence allowing them to build their knowledge and understanding of the diseases in order to be less vulnerable.

Pillar 4: Promote easier and mobile access to health as a form of planning for informal healthier environments. If an outbreak occurs in an ITS the impact will be dramatic due to the lack of equipped facilities and isolation units in remote areas. This also affects host communities and goes beyond the Covid-19 pandemic concerns. Easing restrictions on mobility will facilitate access to food and necessary medicines while promoting mobile and adjustable health services can be seen as a way forward to tackle health and socio-economic vulnerabilities holistically and inclusively.

\section{Conclusion}

While the Covid-19 pandemic is currently contained in Majdal Anjar, and has not spread into other ITSs yet, the situation remains incendiary and can rapidly deteriorate. New community/refugee-led local responses with pandemic-proof strategies for the unplanned are needed in the short term and longer term. Enforced encampment, restriction of mobilities, limited access to health services and extremely poor living conditions expose the refugee population to multiple vulnerabilities and also affect host communities. Critical revisions are required in pre-existing strained relationships between host communities and refugees and in sectors such as shelter and WASH. Changes will rest on the systematic accounting of the local needs of those living in both informal and formal settings to create more inclusive and disease-free environments.

\section{References}

BAKEWELL, O. (2014), 'Encampment and self-settlement', in E. Fiddian-Qasmiyeh, G. Loescher, K. Long and N. Sigona (eds), The Oxford Handbook of Refugee and Forced Migration Studies. Oxford, Oxford University Press, 127-138.

FAWAZ, M. and PEILLEN, I. (2003), The Case of Beirut, Lebanon. Understanding Slums: Case Studies for the Global Report on Human Settlements. London, UCL.

HODALI, D. (2020), Syrian refugees in Lebanon more scared of starvation than COVID19. https://www.dw.com/en/syrian-refugees-in-lebanon-more-scared-of-starvation-thancovid-19/a-53355378 (accessed on 12 May 2020)

THORLEIFSSON, C. (2016), 'The limits of hospitality: coping strategies among displaced Syrians in Lebanon', Third World Quarterly, 37, 1-12. 
TURNER, L. (2015), 'Explaining the (non-)encampment of Syrian refugees: security, class and the labour market in Lebanon and Jordan', Mediterranean Politics, 20, 1-19.

UNHCR (2019), Informal Settlements in Lebanon. UNHRC, n.p.

WILKINSON, A. (2020), 'Local response in health emergencies: key considerations for addressing the COVID-19 pandemic in informal urban settlements', Environment and Urbanisation. https://doi.org/10.1177/0956247820922843 